\title{
Una visión panorámica de la literatura sobre la gestión ambiental en la empresa*
}

\author{
Javier Aguilera Caracuel \\ Universidad Pablo Olavide \\ Natalia Ortiz de Mandojana \\ Universidad de las Islas Baleares
}

\section{Resumen}

La concienciación por minimizar el impacto al entorno natural de las empresas ha adquirido una notable importancia durante las últimas décadas por parte de los diferentes grupos de interés (gobiernos, grupos ecologistas, sindicatos, consumidores, empresas y la sociedad en general). La literatura académica también ha prestado un creciente interés a esta temática. Sin embargo, aún quedan muchos interrogantes respecto a los retos y oportunidades relacionados con la gestión medioambiental. El objetivo del presente trabajo consiste en presentar los principales conceptos y enfoques teóricos utilizados en esta literatura, para resaltar la importancia de la gestión medioambiental de las organizaciones como disciplina académica. En segundo lugar, describimos las diferentes metodologías que se emplean y las fuentes de datos disponibles y, finalmente, proponemos algunas ideas para futuras líneas de investigación.

Palabras clave: gestión medioambiental, stakeholders.

Código JEL: M14, Q10.

\begin{abstract}
Preservation and protection of the natural environment has gained significant attention in the business context, especially during the last decades. Indeed, there are strong pressures from stakeholders (governments, non-governmental organizations, consumers, suppliers and the society) to encourage firms to develop advanced and proactive environmental management practices. Although the management literature has also developed different studies in the environmental arena, there are still many research questions that have to be properly addressed. The aim of this paper is to summarize the main topics covered and the theoretical frameworks used in the existent environmental management literature. Secondly, we describe the main methodologies and databases used in this academic area. Finally, we propose future research lines.
\end{abstract}

Keywords: environmental management, stakeholders

JEL classification: M14, Q10.

* Este trabajo ha sido financiado por el Ministerio de Educación y Ciencia (proyecto ECO201020483) y la Junta de Andalucía (proyectos de excelencia: P08-SEJ-0457, P10-SEJ-6765 y P11SEJ-7988). 


\section{Importancia de la gestión medioambiental de las empresas en el contexto internacional}

La concienciación por minimizar el impacto al entorno natural y la preservación del estado de conservación del planeta han adquirido una notable importancia durante las últimas décadas por parte de los diferentes grupos de interés (stakeholders) como los gobiernos, grupos ecologistas, sindicatos, consumidores, investigadores y la sociedad en general. De hecho, existen importantes llamadas de atención sobre problemas relacionados con el cambio climático, protección de la diversidad, la creación de medidas para prevenir el agotamiento de recursos naturales o los efectos perjudiciales de la contaminación en la salud humana. En relación al ámbito empresarial, hay que tener muy en cuenta que las actividades que ejercen las organizaciones tienen un impacto directo sobre el entorno y, por tanto, sobre el medio ambiente. Por ello, existe una clara necesidad de cambio de actitud por parte de las organizaciones en relación con el medio ambiente (Aguilera Caracuel y Ortiz de Mandojana, 2013).

Siguiendo un orden cronológico, y con objeto de plasmar el interés general sobre la problemática medioambiental, se pueden citar diversos hitos a nivel internacional. En julio de 1972 se celebró en Estocolmo (Suecia) la primera conferencia sobre medio ambiente de las Naciones Unidas, tratándose la problemática medioambiental de forma global aunque aún preliminar. En el año 1985 surge en Canadá una importante iniciativa denominada «Responsible Care». Dicha iniciativa nace con la idea de que las empresas ubicadas en el sector químico, mediante sus asociaciones nacionales, trabajaran conjuntamente con objeto de mejorar los resultados medioambientales y las condiciones de salud y seguridad en la población ${ }^{2}$. De esta forma, mediante el apoyo de asociaciones sectoriales, gobiernos y organizaciones medioambientales no gubernamentales, el mundo empresarial comienza a afrontar paulatinamente el reto medioambiental. Otra muestra de la importancia de la problemática medioambiental se observa cuando la Comisión Mundial para el Medio Ambiente y Desarrollo (WCED), creada en 1984 por la Asamblea General de Naciones Unidas, popularizó el término «desarrollo sostenible» en 1987, definido como «una forma de desarrollo o progreso que satisface las necesidades del presente sin comprometer la capacidad de las futuras generaciones para sostener las suyas propias».

En el año 1992 se celebró la Conferencia de Naciones Unidas sobre medio ambiente y desarrollo sostenible en Río de Janeiro. Dicha cumbre dio lugar a la elaboración del documento «Agenda 21» que supuso el punto de partida para crear una mayor concienciación para la protección medioambiental por parte de gobiernos, empresas, organizaciones no gubernamentales y la población en general. En los siguientes años se han celebrado numerosas cumbres, convenciones, tratados,

${ }^{2}$ Responsible Care es una iniciativa que pretende aplicar prácticas socialmente responsables en la industria química con objeto de contribuir al desarrollo sostenible y a una mejora de las prácticas de gestión medioambiental. Disponible en: http://www.icca-chem.org/en/Home/Responsible-care/Whatwe-do/). 
protocolos y acuerdos entre países para la protección y conservación del medio ambiente. En el año 2001 la Unión Europea (UE) lanzó su IV Programa de Acción en materia de medio ambiente (Medio ambiente 2010: El futuro en nuestras manos) ${ }^{3}$ donde se definían las prioridades y objetivos de la política medioambiental de la UE hasta y después del año 2010, detallando las medidas a adoptar para contribuir a la aplicación de la estrategia de la UE en materia de desarrollo sostenible. En el año 2002, se volvió a celebrar la siguiente «Cumbre de la Tierra» en Johannesburgo («Río $+10 »)$ con el objetivo de que todos los entes sociales continuasen avanzando en importantes temas medioambientales, tales como el Protocolo de Kioto, aprobado en 1997. Dicho protocolo supuso un gran avance puesto que logró tres grandes acuerdos a nivel internacional: (i) la reducción de emisiones de gases a la atmósfera por parte de los gobiernos para evitar el calentamiento global de la Tierra, (ii) una mayor aplicación en todos los ámbitos del concepto de desarrollo sostenible, y (iii) la necesidad de tener en cuenta la expansión poblacional emergente de los nuevos países industrializados cuyo consumo de recursos naturales crece exponencialmente, y plantea serios retos como son la falta de saneamiento o el consumo descontrolado de recursos limitados.

En el año 2007 se llevó a cabo la Cumbre de Bali (Indonesia) con la finalidad de adaptar y redefinir el Protocolo de Kyoto (1997) en base a las nuevas necesidades respecto al cambio climático. En diciembre de 2009 se celebró la XV Cumbre Mundial sobre Cambio Climático en Copenhage (Dinamarca), lográndose establecer compromisos voluntarios entre los distintos países (comprometiendo a las dos principales potencias mundiales: Estados Unidos y China) para reducir las emisiones y el impacto medioambiental de los distintos agentes sociales del entorno. Muy recientemente se han celebrado otras cumbres internacionales de gran calado que han abordado la protección del entorno natural y el desarrollo sostenible, tales como la cumbre de México (2010), Ecuador (2011) y Río de Janeiro (2012).

\section{Principales conceptos y enfoques teóricos de la literatura sobre gestión medioambiental}

La investigación académica sobre la gestión medioambiental de las empresas es aún relativamente reciente. En la década de los 60 se empezó a prestar atención a los problemas que acarreaba la industrialización para la naturaleza, aunque dichos problemas se consideraban de ámbito local. Morrison y Dunlap (1986) consideran que la década de los ochenta es el punto de partida para la expansión de dicha preocupación medioambiental a la sociedad en su conjunto. Después de primeros acercamientos exploratorios, fue en la década de los 90 cuando diversos grupos sociales promovieron un acercamiento más amplio a los problemas ecológicos relacionados

\footnotetext{
${ }^{3}$ Disponible en: http://www.famp.es/recsa/Documentos/4_Medio_Ambiente_2010_El_futuro_esta _en_nuestras_manos/Programa_Medio\%20Ambiente\%202010.pdf.
} 
con la gestión empresarial. El planteamiento inicial pasaba por considerar la creciente preocupación medioambiental como una amenaza para la empresa puesto que debía destinar un gran volumen de recursos para cumplir con la normativa legal, añadiendo costes e incertidumbre en su actividad (Walley y Whitehead, 1994).

Posteriormente, empiezan a surgir los primeros trabajos que analizan de forma detallada la problemática medioambiental, adoptando otro enfoque radicalmente diferente. En este sentido, Porter y van der Linde (1995) hicieron mención expresa al hecho de que las empresas debían empezar a reconocer el medio ambiente como una oportunidad competitiva y no como una amenaza que se podía posponer. Los primeros trabajos en esta nueva corriente se centraron en los procesos medioambientales internos y los recursos (Lawrence y Morrell, 1995), y los programas medioambientales de las organizaciones (Greening, 1995). No obstante, no fue hasta la publicación del número monográfico de la revista Academy of Management Review en el año 1995 sobre «Ecologically sustainable organizations» cuando se consolida la investigación sobre el tema. En referencia a ello fueron piezas teóricas clave los artículos de Hart (1995) y Jennings y Zandbergen (1995).

Por un lado, Hart (1995) enmarca su investigación bajo la perspectiva natural de la teoría de recursos y capacidades. Dicha teoría trata de identificar y explicar las fuentes de una ventaja competitiva sostenible de las organizaciones teniendo en cuenta que los recursos estratégicos están distribuidos heterogéneamente entre las empresas y que las diferencias entre las mismas pueden permanecer en el tiempo (Barney, 1991; Grant, 1991). Posteriormente, muchos autores han abordado la problemática medioambiental bajo el enfoque de recursos y capacidades (Aragón Correa y Sharma, 2003; Christmann, 2000; Marcus y Geffen, 1998; Russo y Fouts, 1997; Sharma, 2000; Sharma y Vredenburg, 1998). Por ejemplo, Sharma y Vredenburg (1998) plantean que la proactividad medioambiental puede llevar a las empresas a desarrollar capacidades únicas y valiosas, tales como la capacidad de integración de los stakeholders, de aprendizaje, innovación y mejora continua. En este sentido, Aragón Correa y Sharma (2003) proponen que la gestión medioambiental proactiva puede actuar como una valiosa capacidad dinámica para la organización que la pone en marcha.

Por otro lado, Jennings y Zandbergen (1995) aplican planteamientos institucionales a la sostenibilidad de las organizaciones. La teoría institucional mantiene la primacía de las fuerzas exógenas en la configuración de las estrategias medioambientales de las empresas (Hoffman, 1999). Entre los temas que se han estudiado bajo este enfoque a lo largo de las últimas décadas, encontramos la posible influencia de las regulaciones medioambientales (Aguilera Caracuel et al., 2010; Aguilera Caracuel et al., 2012; Aguilera Caracuel y Ortiz de Mandojana, 2013; Bansal, 2005; Porter y van der Linde, 1995; Majumdar y Marcus, 2001; Rugman y Verbeke, 1998), entorno institucional de los países y regiones (Aguilera Caracuel et al., 2012b, 2013; Bansal, 2005; Delmas y Montes Sancho, 2011; Hoffmann et al., 2009), la existencia y pertenencia a asociaciones (King y Lenox, 2000), o el nivel de competitividad y cohesión en el sector (Bansal y Roth, 2000). Finalmente, otros trabajos aúnan rasgos 
de la teoría institucional y la de recursos y capacidades mediante el uso de un enfoque contingente (Aguilera Caracuel y Ortiz de Mandojana, 2013; Aragón Correa y Sharma, 2003).

La teoría de los stakeholders también se ha ido configurando como uno de los enfoques principales que ha utilizado la literatura de gestión medioambiental. Freeman (1984:46) define stakeholders como «cualquier persona o grupo de personas que pueda afectar al desempeño de la empresa o que sea afectado por el logro de los objetivos de la organización». Entre los aspectos abordados, se han analizado los diferentes efectos que tiene el desarrollo de la capacidad de integración de los stakeholders en el comportamiento medioambiental de las empresas (Delgado Ceballos et al., 2012; Hart, 1995; Henriques y Sadorsky, 2008; Kassinis y Vafeas, 2002; Rueda Manzanares et al., 2008; Sharma y Vredenburg, 1998). También se ha prestado especial importancia a poder determinar cómo los distintos stakeholders incentivan en mayor o menor medida la adopción de prácticas de gestión medioambiental avanzadas por parte de las empresas (Buysse y Verbeke, 2003). Otros estudios muestran que la presión percibida de cada stakeholders no es igual para todas las organizaciones. En este sentido, Sharma y Henriques (2005) identificaron distintas prácticas medioambientales clasificadas en función del tipo de influencia ejercida por los stakeholders, siendo éstas más o menos proactivas (Aragón Correa, 1998).

La teoría de la dependencia de recursos ha sido también utilizada para analizar la estrategia medioambiental organizativa (Kassinis y Vafeas, 2006; Ortiz de Mandojana et al., 2012). Dicho enfoque sugiere que las organizaciones que dependen de entidades externas para la obtención de recursos críticos son más susceptibles que otras al control y la influencia de dichas entidades (Pfeffer y Salancik, 1978). Por tanto, la mayor dependencia de una organización respecto a un stakeholder, o una entidad externa, implica mayor poder y aumenta la posibilidad de que éste pueda influir en las actividades medioambientales de la organización (Kassinis y Vafeas, 2006; Ortiz de Mandojana et al., 2012).

Junto a ello, Berrone y Gómez Mejía (2009), usando el análisis de los incentivos económicos respaldado por la teoría de la agencia, mostraron que si no se compensa el riesgo y el esfuerzo crecientes asociados a las inversiones medioambientales, los directivos asignan mayor capital a alternativas menos inciertas, manteniendo (o empeorando) el nivel actual de contaminación de la empresa y deteriorando consecuentemente su legitimidad. Por otro lado, Flannery y May (2000), utilizando planteamientos de la teoría del comportamiento planificado de Ajzen (1985), analizaron la influencia de las actitudes de los directivos, el sentimiento de responsabilidad moral, la percepción de control del y la influencia de normas subjetivas sobre el comportamiento medioambiental. Cordano y Frieze (2000) utilizan la misma teoría para analizar las preferencias del comportamiento de los directivos medioambientales. 


\section{Metodologías utilizadas y fuentes de información}

Las aproximaciones al estudio de la gestión medioambiental de las organizaciones son muy diversas. De hecho, numerosos investigadores de varias disciplinas (por ejemplo: dirección estratégica, dirección de operaciones, comportamiento organizativo, entre otras) han abordado el estudio medioambiental de las organizaciones (Etzion, 2007). Como consecuencia de ello, no sólo existe una gran variedad de enfoques teóricos, tal y como hemos visto en la sección anterior, sino que también la problemática abordada es muy compleja y de carácter multidisciplinar.

En primer lugar, atendiendo a los grupos de interés con incidencia en los planteamientos medioambientales de las empresas, la literatura previa ha prestado atención fundamentalmente a los reguladores y poderes públicos (Banerjee, 2001; Hoffman y Ventresca, 1999; Sharma y Henriques, 2005), consumidores (Banerjee, 2001; Christmann, 2004; Hartmann y Apaolaza Ibánez, 2012; Pedersen y Neergaard, 2006), medios de comunicación (Bansal, 2005; Darnall, 2003), inversores (Derwall et al., 2005; Flammer, 2013; Taylor, 2005), grupos activistas (Eesley y Lenox, 2006; Hendry, 2006), consejeros de administración (Berrone y Gómez Mejía, 2009; Bradley et al., 2006; Kassinis y Vafeas, 2002; Ortiz de Mandojana et al., 2012), directivos (Cannella et al., 2008; Walters et al., 2013; Wang y Young, 2013) y la propia cadena de distribución de la empresa (Chung y Weaver, 2013; Wang y Sarkis, 2013; Wong et al., 2012).

Centrándonos en el contexto individual de la organización, numerosos trabajos se han centrado en analizar la innovación en materia medioambiental de las empresas y su efecto en la mejora del desempeño medioambiental, traducido en una reducción significativa de las emisiones de la empresa y un mejor tratamiento y procesamiento de la materia prima y de los residuos (Bocken et al., 2013; Christmann, 2000; Hart, 1995; King y Lenox, 2002; Porter y van der Linde, 1995; Sroufe et al., 2000). La literatura previa muestra que las empresas que llevan a cabo avances e iniciativas medioambientales avanzadas y proactivas (Aragón Correa, 1998) podrán ser capaces de reducir sus costes operativos, mejorando también su desempeño financiero (Hart y Ahuja, 1996). Además, podrán diferenciarse de sus competidores en términos de productos y/o de procesos organizativos (Christmann, 2004; Darnall, 2006). Como consecuencia de ello, las empresas pueden obtener un incremento notable de su reputación y transparencia, así como incrementar su legitimidad gracias a la aprobación social por parte de aquellos stakeholders más concienciados con la problemática medioambiental (Bansal, 2005; Kostova et al., 2008).

Otros trabajos han prestado una importante atención al papel de los propios participantes de la empresa en el proceso de toma de decisiones relacionadas con el medio ambiente (Cordano y Frieze, 2000). En este sentido, numerosos estudios han dado una gran importancia al papel de la alta dirección (Berrone y Gómez Mejía, 2009; Ortiz de Mandoja et al., 2011, 2012). Un buen ejemplo de esta tendencia se plasmó en un monográfico que en el año 2000 publicó la revista especializada Academy of Management Journal, titulado «Management of Organizations in the Natural Environment». Buena parte de los trabajos analizaban la influencia de los directivos 
en la problemática medioambiental (Flannery y May, 2000; Ramus y Steger, 2000; Sharma, 2000). A título ilustrativo, encontramos temáticas que relacionan el liderazgo con el medio ambiente (Andersson y Bateman, 2000; Egri y Herman, 2000), así como el desarrollo de mejores prácticas en dirección medioambiental (Christmann, 2000). Las variables directamente relacionados con los directivos se relacionan con incentivos de tipo económico (Berrone y Gómez Mejía, 2009; Russo y Harrison, 2005), así como con postulados propios de las teorías del comportamiento y consideraciones éticas (Cordano y Frieze, 2000; Flannery y May, 2000).

En cuanto al papel de la dirección de los recursos humanos de la empresa en la formulación e implantación de estrategias medioambientales, existen trabajos que prestan atención al análisis de las percepciones de los miembros de la empresa acerca de la problemática medioambiental (Bansal, 2003; Jian y Bansal, 2003). Otros trabajos muy recientes estudian el papel que juegan los recursos humanos de la empresa en materia de formación medioambiental (Martínez del Río et al., 2012; Vidal Salazar et al., 2012), implantación de normas de certificación medioambiental (Wagner, 2013) y fomento de la mejora en la innovación medioambiental tanto en productos como en procesos (Antonioli et al., 2013).

Otros trabajos se han centrado en analizar el efecto de la presencia de recursos de holgura (slack resources) en la adopción de prácticas medioambientales avanzadas. Los avances en este sentido apuntan en su mayoría a afirmar que aquellas empresas que tienen un volumen sustancial de slack resources tienden a considerar la inversión en medio ambiente como una oportunidad valiosa de negocio, en lugar de una amenaza (Bansal, 2005; Henriques y Sadorsky, 1999; Orlitzky et al., 2003). En esta misma línea, hacemos también mención a todos aquellos estudios que analizan cómo la gestión medioambiental proactiva de las empresas puede repercutir en una mejora sobre su desempeño financiero y organizativo (Aragón Correa et al., 2005; Hart y Ahuja, 1996; Molina Azorín et al., 2009; Russo y Fouts, 1997), siendo los resultados controvertidos y no concluyentes en la literatura (Orlitzky, 2011; Orlitzky et al., 2003). Mientras que hay partidarios que afirman que hay una relación positiva entre desempeño social (incluido el medioambiental) y desempeño financiero de la empresa (Choi y Wang, 2009; Margolis y Walsh, 2001; Waddock y Graves, 1997), otros sostienen que la relación entre ambos es negativa (Barnett 2007; Jensen 2001; Scherer y Palazzo 2011). Finalmente, otra línea de investigación sostiene que no se pueden generalizar los resultados puesto que pueden existir variables moderadoras a tener en cuenta en la citada relación, tales como el tamaño de la empresa, sector de actividad o las propias características el entorno (Orlitzky et al., 2003; Taneja et al., 2011).

Cobran también importancia los estudios que versan sobre el impacto de la internacionalización de las empresas en su estrategia medioambiental (Aguilera Caracuel et al., 2012a, 2012b; Martín Tapia et al., 2010). En este sentido, a pesar de que últimamente se ha analizado la gestión medioambiental de las empresas exportadoras (Aguilera Caracuel et al., 2012b; Martín Tapia et al., 2010), la gran mayoría de trabajos se centra en analizar detenidamente las estrategias medioambientales de 
las empresas multinacionales al ser empresas con una gran repercusión económica y social (Aguilera Caracuel et al., 2011; Kolk y van Tulder, 2001). Concretamente, cobra especial relevancia el estudio de la estrategia de estandarización medioambiental, que conlleva que la empresa multinacional es capaz de establecer una pauta común en su desempeño medioambiental y en sus prácticas medioambientales en las diferentes regiones donde actúa, independientemente del entorno institucional donde opere (Aguilera Caracuel et al., 2010; Christmann, 2004). Dicha estrategia es el resultado de las presiones provenientes de los distintos stakeholders, de la propia industria o de las cooperaciones intergubernamentales (Christmann, 2004; Christmann y Taylor, 2001, 2006), y va más allá del poder coercitivo ejercido por las regulaciones medioambientales de cada país y región. Partiendo de la estrategia de estandarización medioambiental, son numerosos los trabajos que se centran en los antecedentes que conducen a las empresas a adoptar normas de certificación de calidad medioambiental (ISO 14001 o EMAS) y su repercusión final en el desempeño medioambiental, organizativo y financiero (Christmann, 2004; Delmas y Montes Sancho, 2011; Dowell et al., 2000; Montiel y Husted, 2009).

El efecto individualizado ejercido por las legislaciones nacionales e internacionales en el comportamiento medioambiental de las empresas ha sido ampliamente analizado (Marcus et al., 2011; Rugman y Verbeke, 1998). En este sentido, cabe destacar el volumen especial de la revista California Management Review (CMR) en el año 2011, titulado: "Firms, regulatory uncertainty, and the natural environment: What can we expect? What should managers do?». En dicho volumen se recogen importantes trabajos que analizan el impacto de las regulaciones medioambientales en las estrategias organizativas, recogiendo importantes implicaciones para reguladores y poderes públicos (Frank y van Tulder, 2011; Fremeth y Ritcher, 2011).

Resulta también relevante destacar aquellos trabajos que se centran en analizar el flujo de conocimiento medioambiental entre las distintas unidades organizativas de la empresa (Lenox y King, 2004; Wong, 2013) o entre la empresa y agentes externos mediante diferentes mecanismos de comunicación (Geffen y Rothenberg, 2000; Lenox et al., 2000; Wong, 2013).

En cuanto a los sectores de actividad analizados, los primeros trabajos se han centrado en sectores de actividad con gran impacto medioambiental directo, fundamentalmente las industrias química, energética y farmacéutica (King y Lenox, 2002; King y Shaver, 2001). Posteriormente, otros estudios se han centrado en analizar la gestión medioambiental de las empresas de otros sectores de actividad muy relevantes para la economía y con incidencia en el desarrollo sostenible tales como el turismo, principalmente en su dimensión hotelera (López Gamero et al., 2012; Yuan, 2013; Yen y Chen, 2013), aunque también va ganando en importancia el estudio de otros servicios relacionados con la industria turística. No obstante, hay que destacar que, con la excepción del turimo, el número de trabajos que han analizado la gestión medioambiental en el sector servicios es aún escaso.

En cuanto a las fuentes de información medioambiental utilizadas, cabe mencionar que la obtención de información secundaria acerca del desempeño medioam- 
biental de las empresas supone todavía un reto importante debido fundamentalmente a la escasez de datos (Etzion, 2007). En relación a la obtención de datos de desempeño medioambiental (emisiones al aire, agua, tierra y tratamiento y procesamiento de residuos), las bases de datos Toxic Release Inventory (TRI) en Estados Unidos, National Pollutant Release Inventory (NPRI) en Canadá y el Registro Europeo de Emisiones de Sustancias Contaminantes (EPER) en Europa son muy relevantes a nivel internacional. Otros países de Latinoamérica (fundamentalmente México) y de Asia (Corea del Norte y Japón principalmente) están desarrollando actualmente registros medioambientales nacionales donde se recoge el impacto medioambiental de las distintas fábricas pertenecientes a cada empresa.

No obstante, los registros aún se encuentran en una fase preliminar de desarrollo, existiendo escasez de información debido fundamentalmente a la voluntariedad de publicar los datos de desempeño medioambiental (Delgado Ceballos y Rueda Manzanares, 2010). Toda la información está disponible a nivel de fábrica por lo que la agregación de datos a nivel empresa debe hacerse con cautela (King y Shaver, 2001). Son numerosos los trabajos que trabajan con indicadores de desempeño medioambiental provenientes de las bases de datos anteriormente mencionadas (Aguilera Caracuel et al., 2010, 2012a, 2013; King y Lenox, 2000, 2002; King y Shaver, 2001; Russo y Harrison, 2006).

Existen también organismos especializados en la obtención de datos de responsabilidad social corporativa (incluida la información medioambiental), tales como KLD (Kinder, Lydenberg y Domini). Utilizan normalmente una metodología sofisticada por medio de la cual asocian un indicador de desempeño a cada práctica de responsabilidad social corporativa. Para obtener la información, combinan información proveniente de encuestas, entrevistas, rankings de reputación social y medioambiental, memorias detalladas de las propias empresas y noticias relevantes de esa materia publicadas en los últimos años. Numerosos estudios utilizan este tipo de fuente de información (McWilliams y Siegel, 2000; Waddock y Graves, 1997).

Una fuente de información alternativa a KLD es «ASSET4», de Thomson Reuters, que incluye información sobre las prácticas sociales, medioambientales y de gobierno corporativo, constituyendo una herramienta muy útil para inversores con objeto de que puedan incorporar esta información en la evaluación de proyectos. También es posible encontrar información medioambiental en la base de datos Bloomberg, una fuente de información secundaria que provee principalmente de datos económicos y financieros sobre empresas de todo el mundo. Además, incorpora informes medioambientales, sociales y de gobierno corporativo de las empresas.

Concretamente, y con el fin de obtener este tipo de información, Bloomberg utiliza los informes de Responsabilidad Social Corporativa e Informes Anuales publicados por las empresas y una encuesta de sostenibilidad realizada por la propia base de datos. Junto a ello, recopila los datos suministrados por Carbon Diclosure Project (CDP). CDP es una iniciativa privada cuya misión es facilitar el diálogo entre inversores y empresas respecto al cambio climático, posibilitando a las empresas su 
aportación voluntaria de datos con respecto a sus emisiones para entender mejor la evolución de las mismas y comprobar su transparencia. En 2012 Carbon Disclosure Project (CDP) solicitó información a las mayores empresas españolas y portuguesas por capitalización obteniendo un 41 por 100 de ratio de respuesta (CDP, 2012).

Otra alternativa es la presentada por «Sustainalytics» que recoge datos ambientales, sociales y de gobierno corporativo de empresas a nivel global y cuyo principal objetivo es ayudar a inversores institucionales e individuales a identificar inversiones socialmente responsables. Estas fuentes también pueden resultar ser de gran utilidad para investigadores (Orij, 2010; Ortiz de Mandojana et al., 2012).

Finalmente, destacamos aquellos trabajos que utilizan entrevistas y cuestionarios dirigidos directamente a directivos y/o empleados de las empresas con objeto de obtener información medioambiental por medio normalmente de escalas tipo Likert (Aguilera Caracuel et al., 2012b; Martín Tapia et al., 2008). El uso integrado de las diferentes alternativas de información permite sin duda captar la realidad medioambiental de forma más precisa.

Por último, en cuanto al tratamiento metodológico de la información, la gran mayoría de estudios se centra principalmente en recoger la información de una muestra de empresas de determinados países y sectores de actividad. Si bien no todos los resultados son extensibles a la población universal puesto que están contextualizados, se utiliza la lógica de la inferencia estadística. Tanto si se cuenta con datos de corte transversal como longitudinal, la principal técnica estadística utilizada es el modelo de regresión lineal en sus distintas variantes, según la naturaleza de las variables utilizadas.

No obstante, el estudio de caso es un método de estudio muy relevante puesto que permite profundizar en la realidad empresarial y medioambiental de una organización concreta. La información así obtenida puede ser contrastada con la propia opinión de los trabajadores y directivos de la empresa, y enriquecida por medio de la realización de visitas in situ a la propia empresa. No obstante, aún no se ha afianzado esta metodología entre los académicos del área de conocimiento por la creencia de que los resultados obtenidos pueden ser no generalizables al resto de organizaciones.

\section{Futuras líneas de investigación}

Como hemos recogido en las secciones anteriores, la gestión medioambiental se ha asentado claramente como disciplina académica. Muestra de ello, cabe mencionar la inclusión en los últimos años de volúmenes especiales en revistas científicas de gran impacto, que ya han sido citadas anteriormente y a las que se unen otras más recientes publicadas este mismo año, tales como el volumen especial de Management Decision titulado: «Green Management: Fostering sustainable management decisions», o el número especial de la revista Business and Society, denominado: «Corporate sustainability - Off to pastures new or back to the roots? Exploring new perspectives, theories and research methods in sustainability research». 
No obstante, a pesar de la abundante literatura ya existente, que hemos tratado de presentar de forma resumida y ordenar en esa revisión panorámica, aún quedan muchos interrogantes en esta disciplina que suponen retos y oportunidades para realizar nuevas investigaciones. En concreto, nos parecen destacables las siguientes:

1. Considerar la gestión medioambiental como una capacidad dinámica de la empresa en interacción constante con factores internos y del entorno ( $\mathrm{Li}$ y Liu, en imprenta). Esto implica superar el enfoque estático y parcial de la gestión medioambiental y reforzar la naturaleza evolutiva, "path dependent» y sistémica de los aspectos medioambientales. De hecho, en la concepción inicial del término desarrollo sostenible, la Comisión Mundial para el Medio Ambiente y Desarrollo (WCED) promulgaba que el desarrollo industrial debía considerarse conjuntamente con los aspectos sociales y medioambientales para que todo el «sistema» fuera sostenible. Aplicado a nivel de empresa, el concepto de sistema ha ido perdiendo fuerza y los investigadores se han ido centrando en los aspectos económicos, medioambientales y sociales de la empresa de manera separada. La gestión medioambiental ayuda a la empresa a ser sostenible en el sentido que le permite perseguir el triple objetivo social, económico y medioambiental del desarrollo sostenible. La utilización de enfoques integradores y dinámicos ayudan a plasmar este enfoque original de la sostenibilidad.

2. Incluir el aspecto longitudinal como factor esencial para analizar los comportamientos de las variables medioambientales analizadas. La literatura ha llamado la atención crecientemente sobre la necesidad de distinguir los efectos a corto y largo plazo de la gestión medioambiental (López et al., 2007). Pese a ello, los trabajos frecuentemente se centran en analizar las relaciones causaefecto en el corto plazo, normalmente utilizando la perspectiva de un solo año. Esta visión parcial de los efectos de la gestión medioambiental puede llevar a subestimar su potencial para la creación de valor y llevar a decisiones no óptimas respecto a la inversión en la mejora de la gestión medioambiental.

3. La inversión en innovación de los competidores supone un importante reto para las empresas. Por ejemplo, aunque las empresas de Estados Unidos siguen siendo líderes en tecnología solar fotovoltaica, algunas empresas chinas están siendo capaces de reproducir algunos componentes, llegando incluso a dominar algunos desarrollos como la producción de células de silicio cristalino, representando el 65 por 100 de toda la capacidad mundial en 2009 (Haley y Schuler, 2011). La necesidad de proteger los desarrollos tecnológicos se hace evidente, lo que explica que el número de patentes medioambientales haya crecido en los últimos años. El aumento de esta importancia se ha acompañado de un esfuerzo por la identificación de estas patentes. En este sentido, la oficina Europea de Patentes en colaboración con el Programa de Naciones Unidas en Medioambiente (UNEP) y el Centro Internacional en Comercio y Desarrollo Sostenible (ICTSD), han creado el código Y02 que identifica 
patentes relacionadas con la captura de las emisiones de efecto invernadero, la generación y transporte de energía, entre otras. Esto supone una oportunidad para los investigadores que pueden analizar la actividad de patentes de las empresas y sus implicaciones en el área medioambiental. Existen también trabajos que hacen uso de las bases de patentes medioambientales como variable proxy de la innovación medioambiental (Aguilera Caracuel y Ortiz de Mandojana, 2013; Frietsch y Schmoch, 2006).

4. Profundizar en el efecto conjunto de las estructuras de gobierno e internacionalización de las empresas en el diseño de la estrategia medioambiental de las organizaciones. En este sentido, se pueden desarrollar trabajos para tratar de comprender la influencia que ejerce la composición heterogénea y multicultural de los miembros que integran el consejo de administración en la línea de actuación medioambiental por parte de la empresa. También puede arrojarse luz a los factores que conducen a las estructuras de gobierno a considerar ciertas formas de internacionalización (por ejemplo, expansión a través de redes de subsidiarias, exportación, franquicias, alianzas estratégicas o acuerdos de tipo joint-venture) como propulsoras de la puesta en marcha de políticas y prácticas medioambientales proactivas y efectivas por parte de la empresa que mejoren su desempeño, logren reforzar su reputación, legitimidad, y redunden en colaboraciones activas con agentes sociales clave (por ejemplo, gobiernos, reguladores públicos, grupos ecologistas, organizaciones no gubernamentales, consumidores y proveedores).

5. Los mercados de capital determinan la economía global y son un espejo de la evolución de la sociedad. Así, la proliferación a nivel global de los fondos de inversión socialmente responsables (fondos ISR) (Balaguer Franch, 2007) muestra la creciente inclusión en las preferencias de los inversores de objetivos más allá de los meramente económicos pues estos fondos tienen en cuenta la dimensión social, ética y medioambiental de los valores en los que invierten. Como una muestra de este creciente interés, el mercado europeo de ISR experimentó un crecimiento del 102 por 100 entre los años 2005 a 2007 (European Sustainable and Responsible Investment, 2008). Sería interesante profundizar en los efectos que los fondos ISR están teniendo en las empresas en las que invierten, y si su fuerza se ha reducido o reforzado ante la actual crisis económica.

6. Prestar una mayor atención al análisis de la gestión medioambiental de las pequeñas y medianas empresas (PYMES), puesto que ejercen un peso muy importante en el desarrollo sostenible y en el nivel de progreso económico de una región. Además de ello, pueden también tener presencia en mercados extranjeros e interactuar con gran multitud de stakeholders, captando las diferentes demandas medioambientales impuestas por cada grupo de interés.

7. Reforzar el papel de la contabilidad medioambiental como mecanismo de información y control de las buenas prácticas de gestión medioambiental de las empresas. Para ello, resulta vital concienciar a las empresas acerca de la nece- 
sidad de incluir registros de validación con objeto de llevar a cabo auditorías periódicas (Correa Ruiz et al., 2013).

8. Incorporar un mayor volumen de estudios de caso que reflejen la problemática medioambiental particular de determinadas empresas. Aunque los resultados obtenidos no siempre se puedan generalizar, es posible detectar aspectos esenciales a tener muy en cuenta en los modelos de negocio de empresas existentes y también de aquellas empresas de reciente creación.

9. Sería interesante la proliferación de trabajos que utilizaran otras metodologías alternativas (por ejemplo, simulaciones) para comprender mejor los complejos procesos psicológicos y sociales en relación a la gestión medioambiental. Aunque el número de trabajos que utilizan metodologías alternativas es creciente, una mayor variabilidad de enfoques y esfuerzos metodológicos puede ayudar a enriquecer nuestro conocimiento en este campo.

En definitiva, la gestión medioambiental como disciplina académica tiene un amplio y sólido recorrido pero, a su vez, creemos que existen importantes oportunidades y retos para seguir avanzando y profundizando en el conocimiento del que disponemos sobre cómo las empresas afrontan la gestión medioambiental y la minimización de los impactos negativos que su actividad genera.

\section{Referencias bibliográficas}

[1] AGUILERA CARACUEL, J.; ARAGÓN CORREA, J.A. y HURTADO TORRES, N.E. (2010): «El desempeño medioambiental en empresas multinacionales: Influencia de la distancia institucional entre países», Cuadernos de Economía y Dirección de la Empresa, 44 (sept. 2010), pp. 37-58.

[2] AGUILERA CARACUEL, J.; ARAGÓN CORREA, J. A. y HURTADO TORRES, N. E. (2011): «Extending the literature on the environmental strategy of MNEs», Multinational Business Review, 19(4), pp. 299-310.

[3] AGUILERA CARACUEL, J.; ARAGÓN CORREA, J. A.; HURTADO TORRES, N. E. y RUGMAN, A.M. (2012a): «The effects of institutional distance and headquarters' financial performance on the generation of environmental standards in multinational companies», Journal of Business Ethics, 105(4), pp. 461-474.

[4] AGUILERA CARACUEL, J.; HURTADO TORRES, N. E. y ARAGÓN CORREA, J. A. (2012b): «Does international experience help firms to be green? A knowledge based view of how international experience and organisational learning influence proactive environmental strategies», International Business Review, 21(5), pp. 847-861.

[5] AGUILERA CARACUEL, J.; HURTADO TORRES, N. E.; ARAGÓN CORREA, J. A. y RUGMAN, A. M. (2013): «Differentiated effects of formal and informal institutional distance between countries on the environmental performance of multinational enterprises», Journal of Business Research, 66(12), pp. 2657-2665.

[6] AGUILERA CARACUEL, J. y ORTIZ DE MANDOJANA, N. (2013): «Green innovation and financial performance: An institutional approach», Organization \& Environment, 26(4), en imprenta. 
[7] AJZEN, I. (1985): «From intentions to actions: A theory of planned behavior», en J. Kuhl y J. Beckmann (eds.), Action-control: From cognition to behavior, 11-39. Heidelberg: Springer.

[8] ANDERSSON, L. M. y BATEMAN, T.S. (2000): «Individual environmental initiative: Championing natural environmental issues in U.S. business organizations», Academy of Management Journal, 43(4), pp. 548-570.

[9] ANTONIOLI, D; MANCINELLI, S. y MAZZANTI, M. (2013): «Is Environmental Innovation Embedded within High-Performance Organisational Changes? The Role of Human Resource Management and Complementarity in Green Business Strategies», Research Policy, 42(4), pp. 975-988.

[10] ARAGÓN CORREA, J. A. (1998): «Strategic proactivity and firm approach to the natural environment», Academy of Management Journal, 41(5), pp. 556-567.

[11] ARAGÓN CORREA, J. A; GARCÍA MORALES, V. J. y HURTADO TORRES, N. E. (2005): «Un modelo explicativo de las estrategias medioambientales avanzadas para pequeñas y medianas empresas y su influencia en los resultados», Cuadernos de Economía y Dirección de la Empresa, 25, pp. 29-52.

[12] ARAGÓN CORREA, J. A. y SHARMA, S. (2003): «A contingent resource-based view of proactive corporate environmental strategy», Academy of Management Review, 28(1), pp. 71-88.

[13] BALAGUER FRANCH, M. R. (2007): «La inversión socialmente responsable y la responsabilidad social empresarial en los mercados financieros: una aplicación a las instituciones gestoras en España» http://www.cnmv.es/ DocPortal/Publicaciones/MONOGRAFIAS /MON2007_27.pdf

[14] BANERJEE, S. B. (2001): «Managerial implications of corporate environmentalism: Interpretations from industry and strategic implications for organizations, Journal of Management Studies, 38(4), 489-513.

[15] BANSAL, P. (2005): «Evolving sustainably: A longitudinal study of corporate sustainable development», Strategic Management Journal, 26(3), pp. 197-218.

[16] BANSAL, P. (2003): «From issues to action: The importance of individual concerns and organizational values in responding to natural environmental issues», Organization Science, 14(5), pp. 510-527.

[17] BANSAL, P. y ROTH, K. (2000): «Why companies go green: A model of ecological responsiveness», Academy of Management Journal, 43(4), pp. 717-736.

[18] BARNETT, J. (2007): «The geopolitics of climate change», Geography compass, 1(6), pp. 1361-1375.

[19] BARNEY, J. (1991): «Firm resources and sustained competitive advantage», Journal of Management, 17(1), pp. 99-120.

[20] BERRONE, P. y GÓMEZ MEJÍA, L. R. (2009): «Environmental performance and executive compensation: An integrated agency-institutional perspective», Academy of $\mathrm{Ma}$ nagement Journal, 52(1), pp. 103-106.

[21] BOCKEN, N.; MORGAN, D. y EVANS, S. (2013): «Understanding environmental performance variation in manufacturing companies», International Journal of Productivity and Performance Management, 62(8), pp. 856-870

[22] BRADLEY, A; NAGARAJAN, N. J.; SONNENFELD, J. A. y SRINIVASAN, D. (2006): «Does CEO charisma matter? An empirical analysis of the relationship among organizational performance, environmental uncertainty, and top management team perceptions of CEO charisma», Academy of Management Journal, 49(1), pp. 161-174. 
[23] BUYSSE, K. y VERBEKE, A. (2003): «Proactive environmental strategies: A stakeholder management perspective», Strategic Management Journal, 24(5), pp. 453-470.

[24] CANNELLA A, A.; PARK, J. H. y LEE, H. U. (2008): «Top management team functional background diversity and firm performance: Examining the roles of team member co-location and environmental uncertainty». Academy of Management Journal, 51(4), pp. 768-784.

[25] CDP (2012). «Iberia 125 Climate Change Report 2012». Carbon Disclosure Project, disponible en: http://www.ecodes.org/responsabilidad-social/publicaciones-responsabilidad-social/

[26] CHOI, J. y WANG, H. (2009): «Stakeholder relations and the persistence of corporate financial performance», Strategic Management Journal, 30(8), pp. 895-907.

[27] COMISIÓN EUROPEA (2010): «Medio ambiente 2010: El futuro está en nuestras manos», disponible en: http://www.famp.es/recsa/Documentos/4_Medio_Ambiente_2010_ El_futuro_esta_en_nuestras_manos/Programa_Medio\%20Ambiente\%202010.pdf

[28] CORDANO, M. y FRIEZE, I. H. (2000): «Pollution reduction preferences of U.S. environmental managers: Applying Ajzen's theory of planned behavior», Academy of Management Journal, 43(4), pp. 627-641.

[29] CORREA RUIZ, C.; ALBELDA RUIZ, E. y CARRASCO FENECH, F. (2013): «La sostenibilidad y el papel de la contabilidad en la gestión del cambio climático y la ecoinnovación en la Pyme», Cuadernos económicos del ICE, n. ${ }^{\circ}$ 86, diciembre 2013.

[30] CHRISTMANN, P. (2000): «Effects of 'best practices' of environmental management on cost advantage: The role of complementary assets», Academy of Management Journal, 43, pp. 663-680.

[31] CHRISTMANN, P. (2004): «Multinational companies and the natural environment: Determinants of global environmental policy standardization», Academy of Management Journal, 47(5), pp. 747-760.

[32] CHRISTMANN, P. y TAYLOR, G. (2001): «Globalization and the environment: Determinants of firm self-regulation in China», Journal of International Business Studies, 32(3), pp. 439-458.

[33] CHRISTMANN, P. y TAYLOR, G. (2006): «Firm self-regulation through international certifiable standards: Determinants of symbolic versus substantive implementation», Journal of International Business Studies, 37(6), pp. 863-878.

[34] CHUNG, S. H.; WEAVER, R. D. (2013): «Strategic response to pollution taxes in supply chain networks: Dynamic, spatial, and organizational dimensions», European Journal of Operational Research, 231(2), pp. 314-327.

[35] DARNALL, N. (2003): «Why firms adopt ISO 14001: An institutional and resource based view», paper presented at the conference of the Academy of Management, Seattle, WA.

[36] DARNALL, N. (2006): «Why firms mandate ISO 14001 Certification», Business \& Society, 45(3), pp. 354-381.

[37] DELGADO CEBALLOS, J.; ARAGÓN CORREA, J. A.; ORTIZ DE MANDOJANA, N. y RUEDA MANZANARES, A. (2012): «The effect of internal barriers on the connection between stakeholder integration and proactive environmental strategies», Journal of Business Ethics, 107(3), pp. 281-293.

[38] DELGADO CEBALLOS, J. y RUEDA MANZANARES, A. (2010): Public disclosure of corporate environmental performance: Pollutant releases and transfer registers $(P R$ - 
TRs). En J. A. F Stoner y C. Wankel (Series Eds.), Global sustainability as a business imperative (pp. 111-126). New York, NY: Palgrave Macmillan.

[39] DELMAS, M. y MONTES-SANCHO, M. J. (2011): «An institutional perspective on the diffusion of international management system standards: The case of the environmental management standard ISO 14001», Business Ethics Quarterly, 21(1), pp. 103132.

[40] DERWALL, J.; GUENSTER, N.; BAUER, R. y KOEDIJK, K. (2005): «The eco-efficiency premium puzzle«. Financial Analysts Journal, 61(2), pp. 51-63.

[41] DOWELL, G.; HART, S. y YEUNG, B. (2000): «Do corporate global environmental standards create or destroy market value?» Management Science, 46(8), pp. 1059-1074.

[42] EESLEY, C. y LENOX, M.J. (2006): «Firm responses to secondary stakeholder action», Strategic Management Journal, 27, 765-781.

[43] EGRI, C. P. y HERMAN, S. (2000): «Leadership in the North American environmental sector: Values, leadership styles, and contexts of environmental leaders and their organizations», Academy of Management Journal, 43(4), pp. 571-604.

[44] ETZION, D. (2007): «Research on organizations and the natural environment, 1992-Present: A review», Journal of Management, 33(4), pp. 637-664.

[45] EUROPEAN SUSTAINABLE AND RESPONSIBLE INVESTMENT STUDY (2008): http://www.eurosif.org/research/eurosif-sri-study/2008

[46] FLAMMER, C. (2013): «Corporate social responsibility and shareholder reaction: the environmental awareness of investors», Academy of Management Journal, 56(3), 758761.

[47] FLANNERY, B. L. y MAY, D. R. (2000): «Environmental ethical decision making in the U.S. metal-finishing industry», Academy of Management Journal, 43(4), pp. 642662.

[48] FRANK, W. y VAN TULDER, R. (2011): «Integrating environemntal and international strategies in a world of regulatory turbulence», California Management Review, 53(4), pp. 23-46.

[49] FREEMAN, R.E. (1984): Strategic Management: A Stakeholder Approach. Boston: Pitman/Ballinger.

[50] FREMETH, A; RITCHER, B.K. (2011): «Profiting from environmental regulatory uncertainty», California Management Review, 54(1), pp. 145-165.

[51] FRIETSCH, R. y SCHMOCH, U. (2006): «Technological structures and performance as reflected by patent indicators», en U. Schmoch, C. Rammer y H. Legler (eds.), National systems of innovation in comparison: Structure and performance indicators for knowledge societies (pp. 89-105). Dordrecht, Netherlands: Springer.

[52] GEFFEN, C. A. y ROTHENBERG, S. (2000): «Suppliers and environmental innovation: The automotive paint process», International Journal of Operations \& Production Management, 20(2), pp. 166-186.

[53] GRANT, R. M. (1991): «The resource-based theory of competitive advantage: Implications for strategy formulation», California Management Review, 33, pp. 114-135.

[54] GREENING, D.W. (1995): Conservation strategies, firm performance, and corporate reputation in the US electric utility industry. In D. Collins and M. Starik (eds.), Research in corporate social policy and performance-sustaining the natural environment: Empirical studies on the interface between nature and organizations, 345-368. Greenwich, CT: JAI Press. 
[55] HALEY, U. C. V. y SCHULER, D. A. (2011): «Government Policy and Firm Strategy in the Solar Photovoltaic Industry». California Management Review, 54, pp. 17-28.

[56] HART, S. L. (1995): «A natural-resource-based view of the firm», Academy of Management Review, 20(4), pp. 986-1014.

[57] HART, S. L. y AHUJA, G. (1996): «Does it pay to be green? An empirical examination of the relationship between emission reduction and firm performance», Business Strategy and the Environment, 5(1), pp. 30-37

[58] HARTMANN, P. y APAOLAZA IBÁÑEZ, V. (2012): «Consumer attitude and purchase intention toward green energy brands: The roles of psychological benefits and environmental concern», Journal of Business Research, 65(9), pp. 1254-1263.

[59] HENDRY, J. R. (2006): «Taking aim at business: What factors lead environmental non-governmental organizations to target particular firms? Business \& Society, 45(1), 47-86.

[60] HENRIQUES, I. y SADORSKY, P. (1999): «The relationship between environmental commitment and managerial perceptions of stakeholder importance», Journal of Environmental Economics and Management, 42(1), pp. 87-99.

[61] HENRIQUES, I. y SADORSKY, P. (2008): «Oil prices and the stock prices of alternative energy companies», Energy Economics, 30, pp. 998-1010.

[62] HOFFMAN A. (1999): «Institutional evolution and change: environmentalism and the U.S. chemical industry», Academy of Management Journal, 42, pp. 351-372.

[63] HOFFMANN, V. K; TRAUTMAN, T. y HAMPRECHT, J. (2009): «Regulatory uncertainty: A reason to postpone investments? Not necessarily», Journal of Management Studies, 46(7), pp. 1227-1253.

[64] HOFFMAN, A.J. y VENTRESCA, M.J. (1999): « The institutional framing of policy debates: Economics versus the environment, American Behavioral Scientist, 42(8), 1368-1392.

[65] JENNINGS, P. D. y ZANDBERGEN, P. A. (1995): «Ecologically sustainable organizations: An institutional approach», Academy of Management Review, 20, pp. 1015-1052.

[66] JENSEN, M. C. (2001): «Value maximization, stakeholder theory, and the corporate objective function», Business Ethics Quarterly, 12(2), pp. 235-256.

[67] JIAN, R. L. y BANSAL, P. (2003): «Seeing the need for ISO 14001. Journal of Management Studies, 40(4), pp. 1047-1067.

[68] KASSINIS, G. y VAFEAS, N. (2002): »Corporate boards and outside stakeholders as determinants of environmental litigation», Strategic Management Journal, 23(5), pp. 399-415.

[69] KASSINIS, G. y VAFEAS, N. (2006): »Stakeholder pressures and environmental performance», Academy of Management Journal, 49(1), pp. 145-159.

[70] KING, A. A. y LENOX, M. J. (2000): «Industry self-regulation without sanctions: The chemical industry's responsible care program», Academy of Management Journal, 43(4), pp. 698-716.

[71] KING, A. A. y LENOX, M. J. (2002): «Exploring the locus of profitable pollution reduction», Management Science, 48(2), 289-299.

[72] KING, A. A. y SHAVER, J. M. (2001): «Are aliens green? Assessing foreign establishments' environmental conduct in the United States», Strategic Management Journal, 22(11), pp. 1069-1086.

[73] KOLK, A. y VAN TULDER, R. (2010): «International business, corporate social res- 
ponsibility and sustainable development», International Business Review, 39(8), pp. 1359-1378.

[74] KOSTOVA, T.; ROTH, K. y DACIN, M. T. (2008): »Institutional theory in the study of multinational corporations: A critique and new directions», Academy of Management Review, 33(4), pp. 994-1006.

[75] LAWRENCE, A. T. y MORRELL, D. (1995): «Leading-edge environmental management: Motivation, opportunity, resources, and processes», en D. Collins y M. Starik (eds.), Research in corporate social policy and performance-sustaining the natural environment: Empirical studies on the interface between nature and organizations, 99126. Greenwich, CT: JAI Press.

[76] LENOX, M. J. y KING, A. A. (2004): «Prospects for developing absorptive capacity through internal information provision», Strategic Management Journal, 25(4), pp. 331-345.

[77] LENOX, M. J.; KING, A. A. y EHRENFELD, J. R. (2000): «An assessment of design-forenvironment practices in leading U.S. electronics firms». Interfaces, 30(3), pp. 83-94.

[78] LI, D. y LIU, J. (2014): «Dynamic capabilities, environmental dynamism, and competitive advantage: Evidence from China», Journal of Business Research, 67(1), en imprenta.

[79] LÓPEZ, M. V.; GARCÍA, A. y RODRÍGUEZ, L. (2007). «Sustainable development and corporate performance: A study based on the Dow Jones Sustainability Index», Journal of Business Ethics, 75, pp. 285-300.

[80] LÓPEZ GAMERO, M. D.; MOLINA AZORÍN, J. F. y CLAVER CORTÉS, E. (2011): «The relationship between managers' environmental perceptions, environmental management and firm performance in Spanish hotels: A whole framework», International Journal of Tourism Research, 13(2), pp. 141-163.

[81] MAJUMDAR, S. K. y MARCUS, A. A. (2001): «Rules versus discretion: The productivity consequences of flexible regulations», Academy of Management Journal, 44(1), pp. 170-179.

[82] MARCUS, A.; ARAGÓN CORREA, J. A. y PINKSE, J. (2011): «Firms, regulatory uncertainty, and the natural environment», California Management Review, 54(1), pp. $5-16$.

[83] MARCUS, A. y GEFFEN, D. (1998): «The dialectics of competency acquisition: Pollution prevention in electric generation», Strategic Management Journal, 19(12), pp. 1145-1168.

[84] MARGOLIS, J. D. y WALSH, J. P. (2001): People and Profits? The Search for a Link between a Company's Social and Financial Performance. Mahwah, NJ: Lawrence Erlbaum.

[85] MARTÍN TAPIA, I.; ARAGÓN CORREA, J. A. y SENISE BARRIO, M. E. (2008): «Beeing green and export intensity of SMEs: The moderating influence of perceived uncertainty», Ecological Economics, 68(1-2), pp. 56-67.

[86] MARTÍN TAPIA, I.; ARAGÓN CORREA, J. A. y RUEDA MANZANARES, A. (2010): «Environmental strategy and exports in medium, small and micro-enterprises», Journal of World Business, 45(3), pp. 266-275.

[87] MARTÍNEZ DEL RÍO, J.; CÉSPEDES LORENTE, J. y CARMONA MORENO, E. (2012): «High-involvement work practices and environmental capabilities: How HIWPs create environmentally based sustainable competitive advantages», Human Resource Management, 51(6), pp. 827-850 
[88] MCWILLIAMS, A. y SIEGEL D. (2000): «Corporate social responsibility and financial performance: Correlation or misspecification?», Strategic Management Journal, 21(5), pp. 603-609.

[89] MOLINA AZORÍN, J. F.; CLAVER CORTÉS, E ; LÓPEZ GAMERO, M. D. y TARÍ, J. J. (2009): «Green Management and financial performance: A literature review», $M a$ nagement Decision, 47(7), pp. 1080-1100.

[90] MONTIEL, I. y HUSTED, B.W. (2009): «The adoption of voluntary environmental management programs in Mexico: First movers as institutional entrepreneurs», Journal of Business Ethics, 88, pp. 349-363.

[91] MORRISON, D. E. y DUNLAP, R. E. (1986): «Environmentalism and elitism: A conceptual and empirical análisis», Environmental Management, 10, pp. 581-589

[92] ORIJ, RENÉ. (2010): «Corporate social disclosures in the context of national cultures and stakeholder theory» Accounting, Auditing \& Accountability Journal., 23 (7.), pp.868-889.

[93] ORLITZKY, M. (2011): «Institutional logics in the study of organizations: The social construction of the relationship between corporate social and financial performance», Business Ethics Quarterly, 21(3), pp. 409-444.

[94] ORLITZKY, M; SCHMIDT, F.L. y RYNES, S.L. (2003): «Corporate social and financial performance: A meta-analysis», Organization Studies, 24(3), pp. 403-441.

[95] ORTIZ DE MANDOJANA, N.; ARAGÓN CORREA, J. A. y DELGADO CEBALLOS, J. (2011): «La relación entre la propiedad institucional y de los directivos y el desempeño ambiental», Cuadernos de Economía y Dirección de la Empresa, 14 (4), pp. 222-230.

[96] ORTIZ DE MANDOJANA, N.; ARAGÓN CORREA, J. A.; DELGADO CEBALLOS, J. y FERRÓN VILCHEZ, V. (2012): «The Effect of Director Interlocks on Firms' Adoption of Proactive Environmental Strategies», Corporate Governance: An International Review, 20(2), pp. 164-178.

[97] PEDERSEN, E. R. y NEERGAARD, P. (2006): «Caveat emptor-Let the buyer beware! Environmental labelling and the limitations of «green» consumerism». Business Strategy and the Environment, 15, pp. 15-29.

[98] PFEFFER, J. y SALANCIK, G. (1978): The External Control of Organizations. Haper \& Row, New York.

[99] PORTER, M. E. y VAN DER LINDE, C. (1995): «Toward a new conception of the environment-competitiveness relationship», The Journal of Economic Perspectives, 9(4), pp. 97-118.

[100] RAMUS, C. A. y STEGER, U. (2000): «The roles of supervisory support behaviors and environmental policy in employee «ecoinitiatives» at leading-edge European companies», Academy of Management Journal, 43(4), pp. 605-626.

[101] RUEDA MANZANARES, A.; ARAGÓN CORREA, J. A. y SHARMA, S. (2008): «The influence of stakeholders on the environmental strategy of service firms: The moderating effects of complexity, uncertainly and munificence», British Journal of Management, 19, pp. 185-203.

[102] RUGMAN, A. M. y VERBEKE, A. (1998): «Corporate strategies and environmental regulations: An organizing framework», Strategic Management Journal, 19(4), pp. 363-375.

[103] RUSSO, M. V. y FOUTS, P.A. (1997): «A resource-based perspective on corporate en- 
vironmental performance and profitability», Academy of Management Journal, 40(3), pp. 534-559.

[104] RUSSO, M. V. y HARRISON, N. S. (2005): «Organizational design and environmental performance clues from the electronics industry», Academy of Management Journal, 48(4), pp. 582-593.

[105] RUSSO, M. V. y HARRISON, N. S. (2006): «Internal organization and environmental performance: Clues from the electronics industry», Academy of Management Journal, 48(4), pp. 582-593.

[106] SCHERER, A. G. y PALAZZO, G. (2011): «The new political role of business in a globalized world: A review of a new perspective on CSR and its implications for the firm, governance, and democracy», Journal of Management Studies, 48(4), pp. 899-931.

[107] SHARMA, S. (2000): «Managerial interpretations and organizational context as predictors of corporate choice of environmental strategy», Academy of Management Journal, 43(4), pp. 681-697.

[108] SHARMA, S. y HENRIQUES, I. (2005): «Stakeholder influences on sustainability practices in the Canadian forest products industry», Strategic Management Journal, 26(2), pp. 159-180.

[109] SHARMA, S. y VREDENBURG, H. (1998): «Proactive corporate environmental strategy and the development of competitively valuable organizational capabilities», Strategic Management Journal, 19(8), pp. 729-753.

[110] SROUFE, R.; CURKOVIC, S.; MONTABON, F. y MELNYK, S. A. (2000): «The new product design process and design for environment: Crossing the chasm», International Journal of Operations \& Production Management, 20(2), 267-291.

[111] TANEJA, S. S.; TANEJA, P. K. y GUPTA, R. K. (2011): «Researches in corporate social responsibility: A review of shifting focus, paradigms, and methodologies», Journal of Business Ethics, 101(3), pp. 343-364.

[112] TAYLOR, B. (2005): «Consumption: It is time for economists and scientists to talk», Journal of Industrial Ecology, 9(1-2), pp. 14-17.

[113] VIDAL SALAZAR, M. D.; CORDÓN POZO, E. y FERRÓN VÍLCHEZ, V. (2012): «Human resource management and developing proactive environmental strategies: The influence of environmental training and organizational learning», Human Resource Management, 51(6), pp. 905-934.

[114] WADDOCK, S. A. y GRAVES, S. B . (1997): «The corporate social performance-financial performance link», Strategic Management Journal, 18, pp. 303-319.

[115] WAGNER, M. (2013): «Green human resource benefits: Do they matter as determinants of environmental management system implementation?, Journal of Business Ethics, 114(3), pp. 443-456.

[116] WALLEY, N. y WHITEHEAD, B. (1994): «It's not easy being green», Harvard Business Review, 72(3), pp. 46-52.

[117] WANG, X. y YOUNG, M.N. (2013): «Does collectivism affect environmental ethics? A multi-level study of top management teams from chemical firms in China», Journal of Business Ethics, June 5, pp. 1-8.

[118] WANG, Z. y SARKIS, J. (2013): «Investigating the relationship of sustainable supply chain management with corporate financial performance», International Journal of Productivity and Performance Management, 62(8), pp. 871-888.

[119] WALTERS, B; KROLL, M. y WRIGHT, P. (2013): «The impact of top management 
team board member control and environment on post-ipo performance», Academy of Management Journal, 53(3), pp. 572-595.

[120] WONG, S. (2013): «Environmental requirements, knowledge sharing and green innovation: Empirical evidence from the electronics industry in China», Business Strategy and the Environment, 22(5), pp. 321-338.

[121] WONG, C.; LAI, K.; LU, C. y LEUNG, T. (2012) « Green operations and the moderating role of environmental management capability of suppliers on manufacturing firm performance «, International Journal of Production Economics, 140(1), pp. 283-294.

[122] YEN, C. y CHEN, C. (2013): «Perceptions of environmental management and employee job attitudes in hotel firms», Journal of Human Resources in Hospitality \& Tourism, 12(2), pp. 155-174.

[123] YUAN, Y. I. (2013): «Adding environmental sustainability to the management of event tourism», International Journal of Culture, Tourism and Hospitality Research, 7(2), pp. 175-183. 